\title{
CAD Modeling of Coplanar Waveguide Interdigital Capacitor
}

\author{
Nihad Dib, ${ }^{1}$ Jehad Ababneh, ${ }^{1}$ Amjad Omar ${ }^{2}$ \\ ${ }^{1}$ Electrical Engineering Department, Jordan University of Science and Technology, P.O. Box 3030, \\ Irbid 22110, Jordan \\ ${ }^{2}$ Department of Communications Engineering, Hijjawi Faculty of Engineering Technology, Yarmouk \\ University, Irbid, Jordan
}

Received 21 October 2004; accepted 18 January 2005

\begin{abstract}
A lumped-element circuit is proposed to model a coplanar waveguide (CPW) interdigital capacitor (IDC). Closed-form expressions suitable for CAD purposes are given for each element in the circuit. The obtained results for the series capacitance are in good agreement with those available in the literature. In addition, the scattering parameters obtained from the circuit model are compared with those obtained using the full-wave method of moments (MoM) and good agreement is obtained. Moreover, a multilayer feed-forward artificial neural network (ANN) is developed to model the capacitance of the CPW IDC. It is shown that the developed ANN has successfully learned the required task of evaluating the capacitance of the IDC. (c) 2005 Wiley Periodicals, Inc. Int J RF and Microwave CAE 15: 551-559, 2005.
\end{abstract}

Keywords: coplanar waveguide; interdigital capacitor; CAD; neural networks

\section{INTRODUCTION}

Presently, monolithic microwave integrated circuits (MMICs) are increasingly being realized by using the coplanar waveguide (CPW) as the basic transmission line because of its several attractive features [1]. However, in contrast to the well-established microstrip-line technology, there is still a lack of CADoriented models for CPW discontinuities and elements [2].

One of the common elements in microwave circuits is the interdigital capacitor (IDC), which was introduced and analyzed first by Alley [3] almost 35 years ago. Since then, it has been the subject of several studies in the literature [4-14]. Most of these studies concentrated on the design and analysis of microstrip interdigital capacitors. Very few tried to

Correspondence to: N. Dib; email: nihad@just.edu.jo.

DOI 10.1002/mmce.20099

Published online 23 August 2005 in Wiley InterScience (www. interscience.wiley.com). study CPW IDCs [15-17]. A 3D static finite-difference method (FDM) was used in [15] to characterize the CPW IDC and other CPW discontinuities. A $\pi$-network of three capacitors was used to model the CPW IDC. A modified model that consists of $N$ coupled lines in a coplanar environment was used to model the CPW IDC in [16]. Full details of this technique are not available in the literature.

Recently, artificial neural networks (ANNs) have been used in RF and microwave CAD [18-24]. ANNs, sometimes known as the connectionist model, are systems used to model the structure of the brain. This is an attempt to simulate learning based on the observation method used by the brain. The most basic element of the biological model of the brain is called a neuron. The neuron is a specific type of cell which provides humans with the ability to remember, think, and apply previous experiences when taking actions in new situations. In the model, the neuron is a computation element that usually uses a nonlinear function (called the activation function) to process its inputs. 


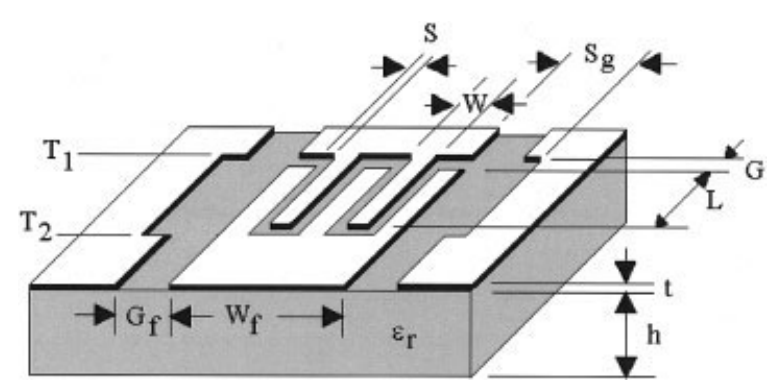

Figure 1. One form of the CPW interdigital capacitor (IDC) with five fingers.

The objective of this article is to study the CPW IDC element. A lumped-element circuit model, which is suitable for computer-aided design (CAD) purposes, is used to model the CPW IDC. The circuit is essentially a $\pi$-network of three capacitors along with a series resistor. Closed-form expressions are given for each element in the circuit. The obtained results for the capacitance and scattering parameters are in good agreement with those available in the literature, and those obtained using the full-wave method of moments (MoM) technique. Moreover, as an alternative to using mathematical expressions, a trained ANN model is developed in order to compute the capacitance of the CPW IDC. The obtained results using the ANN model are very close to those obtained using the mathematical expressions.

\section{EQUIVALENT CIRCUIT MODEL}

One form of the CPW IDC is shown in Figure 1, in which $W$ is the finger width, $S$ is the spacing between fingers, $S_{g}$ is the distance between the outermost fingers and the ground plane, $G$ is the gap width at the end of each finger, $L$ is the overlap (or coupling) length between the fingers, $t$ is the metallization thickness, $h$ is the substrate thickness, $\varepsilon_{r}$ is the dielectric constant of the substrate, $W_{f}$ and $G_{f}$ are the center conductor width and slot width of the feeding CPW, $N$ is the total number of fingers, and $T_{1}$ and $T_{2}$ are the reference planes which coincide with both ends of the CPW IDC.

In Figure 1, the total width of the capacitor is equal to the center conductor width of the feeding CPW. A more general CPW IDC is shown in Figure 2 [16, 17], in which the reference planes will still be at both ends of the IDC, while the rest of the structure (to the left and right of the IDC) can be modeled as CPW transmission-line sections along with CPW step discontinuities.

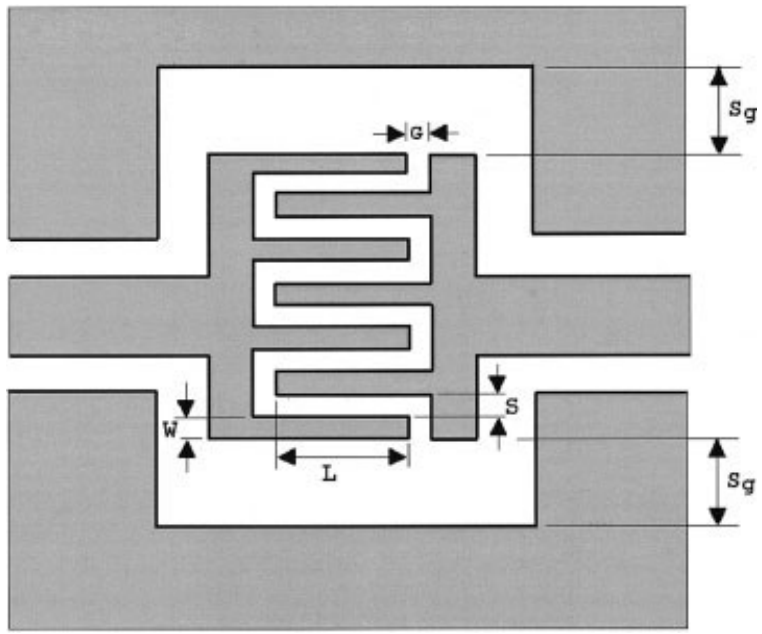

Figure 2. A more general form of the CPW IDC with seven fingers.

The lumped-element equivalent circuit shown in Figure 3 is used to model the CPW IDC [15]. Since a lumped-element model is used, the total size of the IDC should be much smaller than the operating wavelength. Moreover, it should be noted that such a circuit model neglects the series inductance of the IDC, which means that our model should be applicable far below the resonant frequency (for example, $\left.f l f_{\text {res }} \leq 0.2\right)$. Practically, this is usually the frequency range of interest, as it represents the region where the IDC acts as a capacitor with negligible parasitic inductance. As shown in section IV, the model gives very good results up to $15 \mathrm{GHz}$.

In order to account for the metallization thickness $t$, an effective width of the strips can be defined as follows [10]:

$$
W_{e f f}=W+\frac{t}{\pi}\left[1+\ln \left(\frac{4 \pi W}{t}\right)\right] .
$$

In the equations below, the subscript "eff" will be omitted.

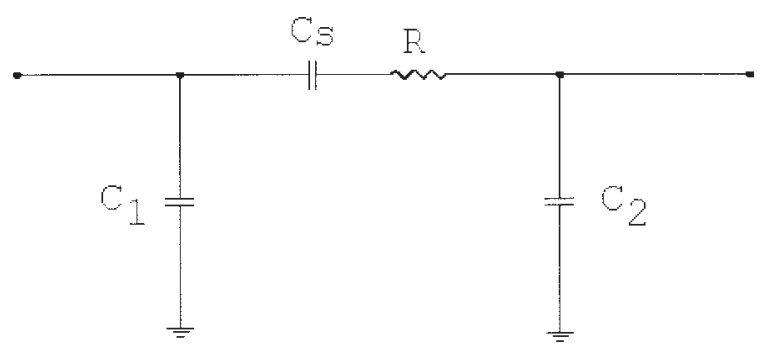

Figure 3. The lumped-element equivalent circuit of the CPW IDC. 
The lumped elements of this circuit are obtained as follows.

\section{A. Capacitance $C_{s}$}

Capacitance $C_{s}$ is the most important element in the circuit, and has been the subject of study in many papers [3-7]. Recently, using conformal mapping, Gevorgian [10] presented a closed-form expression for the $C_{s}$ of a multilayered substrate IDC. For a single-layered substrate, which is the case under consideration, the following expression can be used:

$$
C_{s}=C_{3}+C_{N}+C_{e n d},
$$

where $C_{3}$ is the capacitance of a three-finger IDC, $C_{N}$ is the capacitance of a periodical $(N-3)$ structure, and $C_{\text {end }}$ is the capacitance due to the open ends at the end of each finger. The following expression is used to evaluate $C_{3}$ [10]:

$$
\begin{gathered}
C_{3}=4 \varepsilon_{0} \varepsilon_{e} \frac{K\left(k_{1}\right)}{K\left(k_{1}^{\prime}\right)} L, \\
\varepsilon_{e}=1+q_{1} \frac{\varepsilon_{r}-1}{2}, \\
q_{1}=\frac{K\left(k_{1}^{\prime}\right) \frac{K\left(k_{2}\right)}{K\left(k_{1}\right)}}{K\left(k_{2}^{\prime}\right)} \\
k_{2}=\frac{k_{1}=\frac{W}{W+2 S} \sqrt{\left.\frac{W}{3 W+2 S}\right]^{2}}}{1-\left[\frac{W}{3 W+2 S}\right]^{2}}, \\
\frac{\sinh \left(\frac{\pi(W+2 S)}{\sinh \left(\frac{\pi W}{4 h}\right)}\right.}{\sinh ^{2}\left(\frac{\pi(3 W+2 S)}{4 h}\right)-\sinh ^{2}\left(\frac{\pi(W+2 S)}{4 h}\right)}
\end{gathered}
$$

where $k_{i}^{\prime}=\sqrt{1-k_{i}^{2}}$ and $K(k)$ is the complete elliptic integral of the $1^{\text {st }}$ kind. The ratio $K(k) / K\left(k^{\prime}\right)$ can be evaluated using the well-known Hilberg's approximate expressions [25]. It should be noted that there is a misprint in eq. (15) in [10], which has been taken care of in the above equations.
Similarly, the following expression is used to evaluate $C_{N}[10]$ :

$$
\begin{gathered}
C_{N}=(N-3) \varepsilon_{0} \varepsilon_{N} \frac{K\left(k_{3}\right)}{K\left(k_{3}^{\prime}\right)} L, \\
\varepsilon_{N}=1+q_{N} \frac{\varepsilon_{r}-1}{2}, \\
q_{N}=\frac{K\left(k_{3}^{\prime}\right)}{K\left(k_{3}\right)} \frac{K\left(k_{4}\right)}{K\left(k_{4}^{\prime}\right)},
\end{gathered}
$$

$$
k_{3}=\frac{W}{W+S}
$$

$$
\begin{aligned}
& k_{4}= \frac{\sinh \left(\frac{\pi W}{4 h}\right)}{\sinh \left(\frac{\pi(W+S)}{4 h}\right)} \\
& \times \sqrt{\frac{\cosh ^{2}\left(\frac{\pi(W+S)}{4 h}\right)+\sinh ^{2}\left(\frac{\pi(W+S)}{4 h}\right)}{\cosh ^{2}\left(\frac{\pi W}{4 h}\right)+\sinh ^{2}\left(\frac{\pi(W+S)}{4 h}\right)}} .
\end{aligned}
$$

An expression for $C_{\text {end }}$ is also derived using conformal mapping in [10]. Using the $C_{\text {end }}$ expression given in [10], we were not able to reproduce the results presented in the paper itself. There seems to be something wrong in the presented expression for $C_{\text {end }}$ [26]. Thus, the model presented in [2] is used to compute $C_{\text {end }}$, which relies on the simple expressions given in $[27,28]$. This is accomplished by evaluating the openend capacitance of a CPW with center-conductor width $W$, slot width $S$, and end-gap width $G$. Then, this is multiplied by the number of fingers $N$, since we have $N$ open ends. To obtain the CPW open-end capacitance, the expression derived in [28], based on the assumption of narrow slots, is used when $G /(S+$ $2 W) \leq 0.2$, while the simple formula for the effective length extension $\left(\ell_{o c}\right)$ given in [27] is used otherwise.

Finally, in the case of two fingers $(N=2)$, a structure with two strips exists, which is essentially the coplanar-strips (CPS) line [1]. The capacitance of the CPS can be easily obtained from that of the CPW using the duality principle [1]. So, the total capacitance $C_{s}$ of a two-finger IDC is given by

$$
C_{s}=C_{\text {end }}+L C_{C P S}
$$




\section{B. Resistance $R$}

Resistance $R$ is computed using the expression in [3], which is given by

$$
R=\frac{4}{3} \frac{L R_{s}}{N W},
$$

where $R_{s}$ is the surface resistance. Usually, $R$ is very small and has a negligible effect on the response of the IDC.

\section{Capacitors $C_{1}$ and $C_{2}$}

Parallel capacitors $C_{1}$ and $C_{2}$ are used to model the capacitance between the IDC (mainly the outermost fingers) and the ground planes. In Figures 1 and $2, S_{g}$ is the distance between the outermost finger of the IDC and the ground planes. Usually, this distance is chosen to be relatively large so as to reduce the ground effect on the IDC response. However, the ground effect needs to be included in order to obtain more accurate results. To our knowledge, there are no expressions available in the literature to evaluate $C_{1}$ and $C_{2}$. An approximate expression can be obtained as explained below.

Note that the outermost finger and the ground plane beside it constitute a CPW line with one ground plane only (instead of two in the conventional CPW). This asymmetric CPW has strip width $W$ and slot width $S_{g}$. Such a structure has been studied in [1, 29], and its capacitance $C_{C P W 1}$ per unit length is given by

$$
\begin{gathered}
C_{C P W 1}=2 \varepsilon_{0} \frac{K\left(k_{5}^{\prime}\right)}{K\left(k_{5}\right)}+\varepsilon_{0}\left(\varepsilon_{r}-1\right) \frac{K\left(k_{6}\right)}{K\left(k_{6}^{\prime}\right)}, \\
k_{5}=\sqrt{\frac{S_{g}}{W+S_{g}}}, \\
k_{6}=\sqrt{\frac{e^{\pi W / h}-1}{e^{\pi\left(W+S_{g}\right) / h}-1}} .
\end{gathered}
$$

It should be noted that when $N$ is odd, the number of fingers connected to one of the ports exceeds the number of those connected to the other port by one. Thus, the outermost two fingers will be connected to the same port (for example, port 1). In this case, one of the shunt capacitances will be computed as follows:

$$
C_{1}=2 C_{C P W 1}(L+G) \text {. }
$$

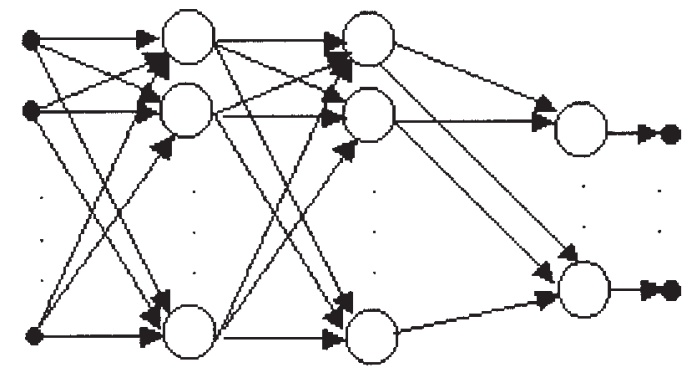

Input Layer Hidden Layers

Output Layer

Figure 4. Example of a multilayer feed-forward network architecture (an input layer, two hidden layers, and an output layer).

Regarding $C_{2}$, the results presented in [15] show that for odd $N, C_{1}$ is almost double $C_{2}$. Thus, in our circuit model, it will be assumed that $C_{2}=C_{1} / 2$ for odd $N$.

On the other hand, when $N$ is even, one of the outermost fingers will be connected to port 1 , while the other finger will be connected to port 2 . In this case, the following equation will be used to evaluate the shunt capacitances:

$$
C_{2}=C_{1}=C_{C P W 1}(L+G)
$$

The above expressions neglect the capacitance between the inner fingers and the ground. In addition, it should be noted that when $N$ is even, a symmetric structure exists (that is, the phase of $S_{11}=$ the phase of $S_{22}$ ), while when $N$ is odd, the phases of $S_{11}$ and $S_{22}$ are different.

\section{ANN Modeling of $C_{s}$}

The multilayer feed-forward network is one of the most effective and commonly used ANN architectures [30]. In this architecture, the neurons are arranged in layers, as shown in Figure 4. The network consists of an input layer, output layer, and a number of what is called hidden layers (Fig. 4 shows an input layer, two hidden layers, and an output layer).

The feed-forward network got its name from the fact that the output of a given layer is forwarded as input to the following layer (starting with the input layer and ending with the output layer). A trial-anderror or simple-to-complex procedure is used to determine the network architecture for a given problem. However, the number of hidden layers to be used depends on the complexity of the task for which the network is to be trained. In addition, the degree of nonlinearity of the input-output mapping required from the network affects the number of neurons and 
TABLE I. The Range of Input Parameters

\begin{tabular}{lccccc}
\hline$\varepsilon_{r}$ & $h[\mu \mathrm{m}]$ & $W[\mu \mathrm{m}]$ & $S[\mu \mathrm{m}]$ & $t[\mu \mathrm{m}]$ & $G[\mu \mathrm{m}]$ \\
\hline $9-13$ & $100-700$ & $10-50$ & $2-50$ & $1-5$ & $2-50$ \\
\hline
\end{tabular}

the type of activation function to be used in each layer. The most commonly used activation function with a feed-forward network is the sigmoid function, given by

$$
\sigma(n)=\frac{1}{1+e^{-n}}
$$

Training is a very important issue in developing an ANN to perform a certain task. By training, we mean the adjustment of the weights of each layer such that the output of a network will be as close as possible to a required or targeted output. Back-propagation is the most common algorithm used to train a feed-forward network [31]. It is a gradient-decent algorithm, in which the network weights are moved along the negative of the gradient of a given performance function. The mean-squared error is normally used as a performance function while training a feed-forward network.

In this article, as an alternative to using mathematical expressions, a multilayer feed-forward network is developed to model the capacitance $C_{s}$ of the CPW IDC. We have used the parameters: $\varepsilon_{r}, h, W, S, t$, and $G$ of the CPW IDC as the input to the network and $C_{3} /\left(L \varepsilon_{0}\right), C_{N} /\left(L(N-3) \varepsilon_{0}\right)$, and $C_{\text {end }} / N$ as the output of the network. Then, the value of $C_{s}$ for some given values of $N$ and $L$ can be obtained directly using eq. (2). Therefore, our network needs to have three neurons in the output layer that represent $C_{3} /\left(L \varepsilon_{0}\right), C_{N} /(L$ $\left.(N-3) \varepsilon_{0}\right)$, and $C_{\text {end }} / N$. Eq. (2) was used to create data for training and testing the network. The range of the input parameters used in designing the data is given in Table I. This range covers most practical CPW IDCs used in MMICs.

A training set of 1000 randomly distributed points of the parameters in the range given in Table I and the back-propagation training algorithm were used to
TABLE II. The Accuracy of the Developed ANN

\begin{tabular}{llll}
\hline & $\begin{array}{c}C_{3} / \\
\left(L \varepsilon_{0}\right)\end{array}$ & $\begin{array}{c}C_{N} / \\
\left(L \varepsilon_{0}(N-3)\right)\end{array}$ & $C_{\text {end }} / N$ \\
\hline Training & & & \\
\hline $\begin{array}{l}\text { Average error } \\
\quad \text { Standard deviation }\end{array}$ & 0.0004 & 0.0000492 & 0.000879 \\
$\begin{array}{l}\text { Testing } \\
\text { Average error }\end{array}$ & 0.0008 & 0.0019 & 0.0976 \\
$\quad$ Standard deviation & 0.0929 & 0.032 & 0.0299 \\
\hline
\end{tabular}

train the neural network. On the other hand, we used 100 completely different, randomly chosen cases to test the network. Both the training and testing data were linearly normalized to have values between 0 and 1. It was found that a network of two hidden layers (with 15 neurons in the first and 25 neurons in the second) can successfully model the capacitance $C_{s}$. The accuracy of the trained network with this architecture is given in Table II in terms of average error and standard deviation. Therefore, for a given set of input parameters, the value of $C_{s}$ can be accurately computed in negligible time using the developed ANN.

\section{RESULTS}

The first comparison was made with the values of $C_{s}$ presented in [10]. Tables III and IV show some comparisons. Although we used the same expressions presented in [10] (except for the evaluation of $C_{\text {end }}$ ), slightly different results are obtained. In Tables III and IV, the results obtained using the expression given in [32] are also included and are in very good agreement with our results. No reference (or detail) is given about this expression in [32], but it seems to be obtained using conformal mapping as well (it will be referred to as Gupta's expression). It can be clearly seen that the ANN model gives very close results to those obtained by eq. (2). This shows that the developed ANN has successfully learned the required task of evaluating the capacitance $C_{s}$ of the IDC.

TABLE III. $h=600 \mu \mathrm{m}, \varepsilon_{r}=12.5, S=W=G=10 \mu \mathrm{m}, t=5 \mu \mathrm{m}, L=100 \mu \mathrm{m}\left(C_{s}\right.$ in pF)

\begin{tabular}{llllll}
\hline$N$ & \multicolumn{1}{c}{5} & \multicolumn{1}{c}{10} & \multicolumn{1}{c}{20} & \multicolumn{1}{c}{50} & 205 \\
\hline$C_{s}$, theory [6] & 0.036 & 0.079 & 0.165 & 0.425 & 1.215 \\
$C_{s}$, experiment [6] & 0.055 & 0.09 & 0.16 & 0.5 & \\
$C_{s}$ [10] & 0.0475 & 0.0945 & 0.183 & 0.453 & 1.86 \\
$C_{s}$ [32] & 0.029 & 0.069 & 0.136 & 0.351 & 1.46 \\
$C_{s}$, eq. (2) & 0.0331 & 0.0658 & 0.1313 & 0.3276 & 1.342 \\
$C_{s}$, ANN & 0.0336 & 0.0669 & 0.1335 & 0.3334 & 1.366 \\
\hline
\end{tabular}


TABLE IV. $h=101 \mu \mathrm{m}, \varepsilon_{r}=12.5, t=1.5 \mu \mathrm{m}$. CAP1: $N=10, L=254 \mu \mathrm{m}, W=20 \mu \mathrm{m}, S=G=$ $4.8 \mu \mathrm{m}$. CAP2: $N=19, L=340 \mu \mathrm{m}, W=12 \mu \mathrm{m}$, $S=G=4.25 \mu \mathrm{m}\left(C_{s}\right.$ in $\left.\mathrm{pF}\right)$

\begin{tabular}{lcc}
\hline & $C_{s}$, CAP1 & $C_{s}$, CAP2 \\
\hline Measured [5] & 0.247 & 0.607 \\
Computed [5] & 0.242 & 0.59 \\
[10] & 0.283 & 0.587 \\
[32] & 0.238 & 0.55 \\
Eq. (2) & 0.2033 & 0.4481 \\
ANN & 0.2039 & 0.4483 \\
\hline
\end{tabular}

Although Tables III and IV show some variation between our results and those available in the literature, the other comparisons (presented below) give us more confidence in our results. It is believed that the experimental results in $[5,6]$ are not very accurate, since they rely upon wire bonds and resonance measurement, not upon the direct measurement of the component with modern de-embedding techniques. Moreover, it should be noted that a microstrip IDC was considered in $[5,6]$ and the effect of the terminal strips of the IDC was included in the measurements and the analysis. This explains the higher capacitance values obtained in $[5,6]$, as compared to our results and those obtained using the expression from [32].

The second comparison was made with the results presented in [15], in which the quasi-static FDM was used to obtain the series capacitance $C_{s}$ along with the shunt capacitances $C_{1}$ and $C_{2}$ of the CPW IDC of the type shown in Figure 1 with $S_{g}=G_{f}$. Our results and those obtained using Gupta's expression [32] are shown in Table $\mathrm{V}$ in comparison with those presented in [15]. Our results are in very good agreement with the FDM ones, except for the second case (CAP2), which has a very small $S$ of $2 \mu \mathrm{m}$, while the thickness $t$ is $3 \mu \mathrm{m}$ ! Unfortunately, the distance $S_{g}$, which is needed to compute $C_{1}$ and $C_{2}$, is not given in [15], and thus, no comparison can be made for the shunt capacitances. However, $S_{g}=52 \mu \mathrm{m}$, which results in a $50 \Omega$ feeding line for the first case (CAP1), was tried and hence $C_{1}=C_{2}=9.1 \mathrm{fF}$ was obtained, as compared to
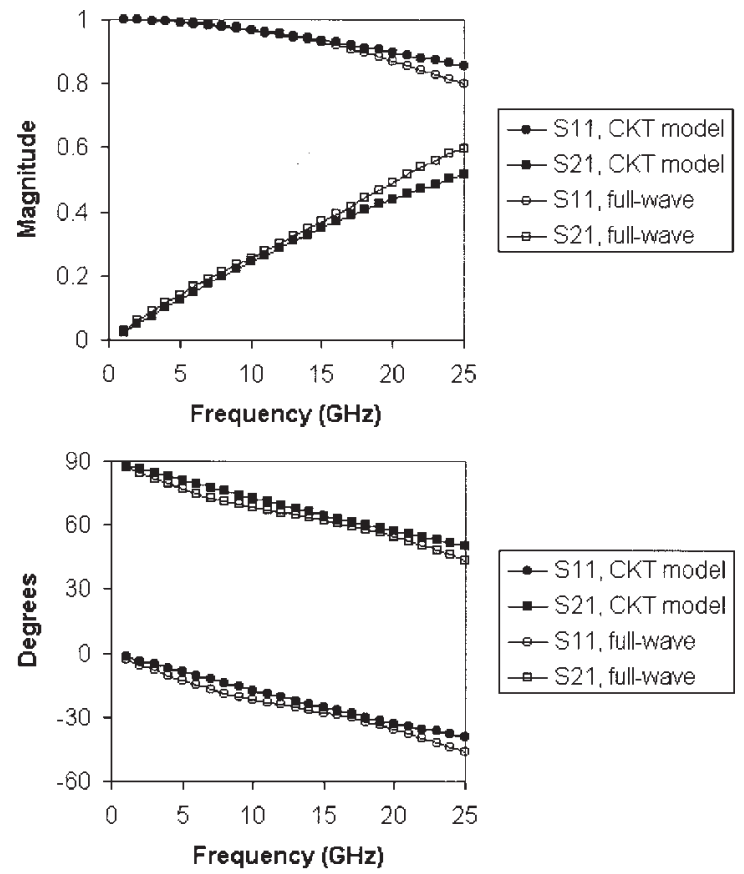

Figure 5. $S$-parameters of the CPW IDC (CAP1 in Table V).

$9.65 \mathrm{fF}$ using FDM [15]. In addition, $S_{g}=125 \mu \mathrm{m}$, which results in a $50 \Omega$ feeding line for the fourth case (CAP4), was tried and hence $C_{1}=27.3 \mathrm{fF}$ was obtained, as compared to $23 \mathrm{fF}$ using FDM [15].

The scattering parameters for the first (CAP1), third (CAP3), and fourth (CAP4) cases obtained using the circuit (CKT) model are shown in Figures 5-7. Full-wave results obtained using the software IE3D are also shown in the same figures. IE3D is a fullwave, MoM-based electromagnetic simulator [33]. As expected, the difference between the results of the full-wave and CKT models increases as the frequency increases, since quasi-static conditions were assumed when deriving the expressions for the lumped elements of the circuit model (that is, the values of the equivalent capacitances are independent of frequency). Good agreement is obtained up to $15 \mathrm{GHz}$. This conforms to the conclusion in [10] that the conformal-mapping model for $C_{s}$ can be used for frequen-

TABLE V. Comparison with Results from [15] $\left(C_{s}\right.$ in $\mathrm{fF}$, dimensions in $\left.\mu \mathrm{m}\right)$

\begin{tabular}{|c|c|c|c|c|c|c|c|c|c|c|c|c|c|}
\hline & $\varepsilon_{r}$ & $N$ & $W$ & $S$ & $G$ & $t$ & $h$ & $L$ & $\begin{array}{c}C_{s}, \\
\text { FDM } \\
{[15]}\end{array}$ & $\begin{array}{l}C_{s} \\
\text { exp. } \\
{[15]}\end{array}$ & $\begin{array}{c}C_{s} \\
{[32]}\end{array}$ & $\begin{array}{l}C_{s} \text {, } \\
\text { eq. } \\
(2)\end{array}$ & $\begin{array}{c}C_{s}, \\
\text { ANN }\end{array}$ \\
\hline CAP1 & 12.9 & 4 & 17 & 3 & 3 & 3 & 400 & 97 & 40.1 & 41 & 34.8 & 40.4 & 40.5 \\
\hline CAP2 & 12.9 & 10 & 12 & 2 & 2 & 3 & 400 & 97 & 113.3 & 116 & 109 & 96.4 & 95.9 \\
\hline CAP3 & 12.9 & 4 & 17 & 3 & 3 & 3 & 400 & 197 & 73 & 76 & 71 & 74.8 & 75.2 \\
\hline CAP4 & 9.8 & 5 & 38 & 25 & 25 & 5 & 635 & 175 & 55 & 56 & 42 & 54.8 & 54.8 \\
\hline CAP5 & 9.8 & 7 & 38 & 25 & 25 & 5 & 635 & 175 & 85 & 86 & 63 & 76.5 & 76.5 \\
\hline
\end{tabular}



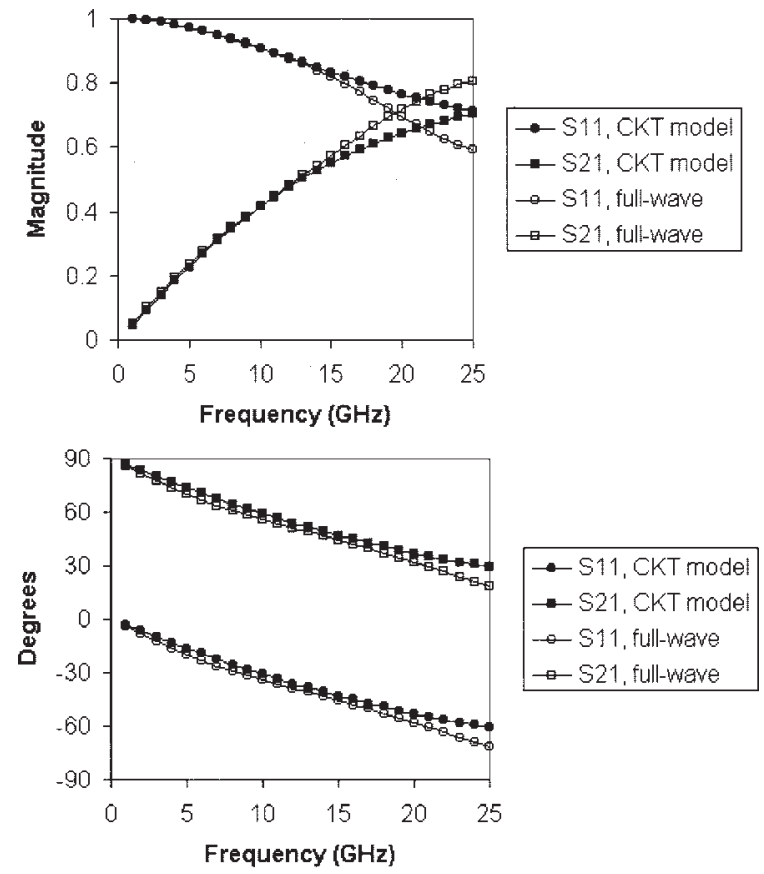

Figure 6. $S$-parameters of the CPW IDC (CAP3 in Table V).

cies up to the X-band. The agreement in the case of $N=4$ (Figs. 5 and 6) is better than the case with $N=$ 5 (Fig. 7). This might be due to the assumption of $C_{2}=C_{1} / 2$ for odd $N$, which may not be exact.

The microstrip IDC has been experimentally studied in [7], where an equivalent circuit similar to the
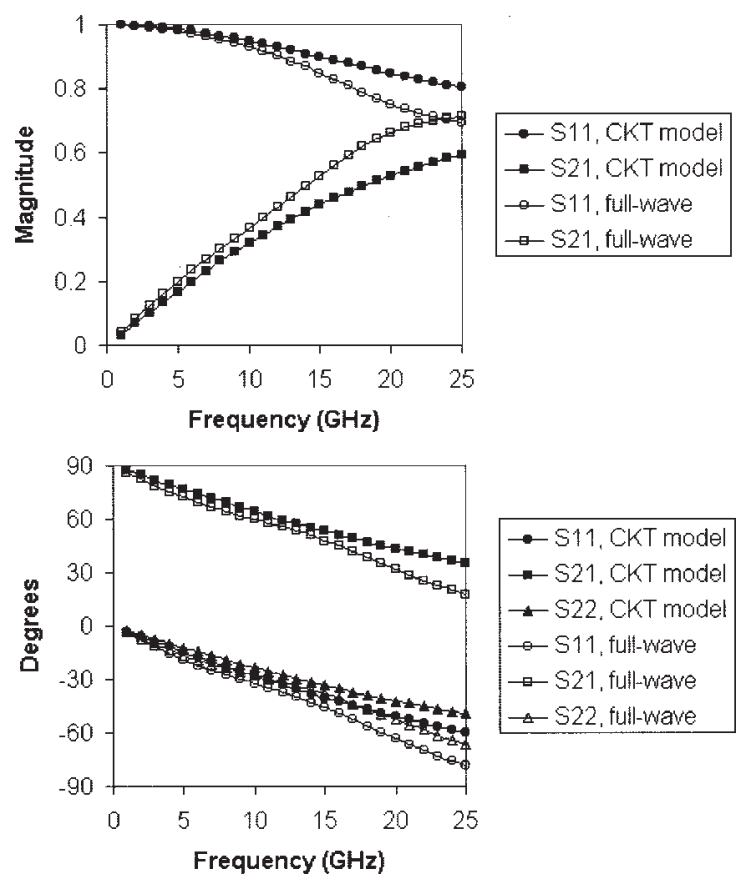

Figure 7. $S$-parameters of the CPW IDC (CAP4 in Table V).
TABLE VI. Comparison with the Results From [7]. $h=125 \mu \mathrm{m}, \varepsilon_{r}=13, W=G=12 \mu \mathrm{m}, S=8 \mu \mathrm{m}$, $t=5 \mu \mathrm{m}, N=20\left(C_{s}\right.$ in pF)

\begin{tabular}{lccll}
\hline$L(\mu \mathrm{m})$ & \multicolumn{1}{c}{80} & \multicolumn{1}{c}{180} & \multicolumn{1}{c}{300} & \multicolumn{1}{c}{400} \\
\hline$C_{s}[7]$ & 0.126 & 0.252 & 0.405 & 0.527 \\
$C_{s}[32]$ & 0.128 & 0.287 & 0.48 & 0.64 \\
$C_{s}$, eq. (2) & 0.125 & 0.25 & 0.399 & 0.523 \\
$C_{s}$, ANN & 0.1245 & 0.2488 & 0.398 & 0.5224 \\
\hline
\end{tabular}

one presented here was used to model the IDC. What concerns us here is the series capacitance $C_{s}$. Table VI shows our results in comparison to those found experimentally in [7]. The results using Gupta's expression are also shown. It can be seen that our results are in very good agreement with those in [7], although a microstrip IDC is considered in [7]. This is due to the fact that the substrate thickness is $125 \mu \mathrm{m}$, which makes the effect of the bottom ground plane (existing in the microstrip IDC) on $C_{s}$ rather small.

\section{CONCLUSION}

A lumped-element equivalent circuit, which is suitable for CAD purposes, has been used to model a CPW IDC. The circuit is essentially a $\pi$-network consisting of three capacitors. Expressions for each element in the circuit model have been given and studied. Although Tables III and IV show some variation between our results and those available in the literature, Tables V and VI (and Figs. 5-7) give us more confidence in our expressions.

Moreover, a multilayer feed-forward ANN has been developed to model the capacitance of the CPW IDC. It was shown that the developed ANN successfully learned the required task of evaluating the capacitance of the IDC. At the present time, the developed ANN model, along with an optimization method, is being used to design CPW interdigital capacitors (similar to the work done on microstrip IDCs in [22]); that is, given a specific capacitance value $C_{s}$, the dimensions of the IDC and the substrate parameters can be obtained.

\section{REFERENCES}

1. R. Simons, Coplanar waveguide circuits, components, and systems, Wiley, New York, 2001.

2. N. Dib, A comprehensive study of CAD models of several coplanar waveguide (CPW) discontinuities, IEE Proc Microwaves Antennas Propagat 152 (2005), 6976. 
3. G. Alley, Interdigital capacitors and their application to lumped-element microwave-integrated circuits, IEEE Trans Microwave Theory Tech 18 (1970), 1028-1033.

4. J. Hobdell, Optimization of interdigital capacitors, IEEE Trans Microwave Theory Tech 27 (1979), 788 791.

5. R. Esfandiari, D. Maki, and M. Siracusa, Design of interdigital capacitors and their application to gallium arsenide monolithic filters, IEEE Trans Microwave Theory Tech 31 (1983), 57-64.

6. E. Pettenpaul, H. Kapusta, A. Weisgerber, H. Mampe, J. Luginsland, and I. Wolff, CAD models of lumped elements on GaAs up to $18 \mathrm{GHz}$, IEEE Trans Microwave Theory Tech 36 (1988), 294-304.

7. V. Sadhir, I. Bahl, and D. Willems, CAD-compatible accurate models of microwave passive elements for MMIC applications, Int J Microwave and MillimeterWave CAE 4 (1994), 148-162.

8. H. Wu, Z. Zhang, F. Barnes, C. Jackson, A. Kain, and J. Cuchiaro, Voltage-tunable capacitors using hightemperature supercondcutors and ferroelectrics, IEEE Trans Appl Superconductivity 4 (1994), 156-160.

9. C. Chi and G. Rebeiz, Planar microwave and millimeter-wave lumped elements and coupled-line filters using micro-machining techniques, IEEE Trans Microwave Theory Tech 43 (1995), 730-738.

10. S. Gevorgian, T. Martinsson, P. Linner, and E. Kolberg, CAD models for multilayered substrate interdigital capacitors, IEEE Trans Microwave Theory Tech 44 (1996), 896-904.

11. G. Coen, D. De Zutter, and N. Fache, Automatic derivation of equivalent Circuits for general microstrip interconnection discontinuities, IEEE Trans Microwave Theory Tech 44 (1996), 1010-1016.

12. L. Zhu and K. Wu, Accurate circuit model of an interdigital capacitor and its application to the design of new quasi-lumped miniaturized filters with suppression of harmonic resonance, IEEE Trans Microwave Theory Tech 48 (2000), 347-356.

13. D. Caratelli and R. Cicchetti, A full-wave analysis of interdigital capacitors for planar integrated circuits, IEEE Trans Magn 39 (2003), 1598-1601.

14. N. Dib, Q. Zhang, and U. Rohde, New CAD model of the microstrip interdigital capacitor, Active and Passive Electron Compon 27 (2004), 237-245.

15. M. Naghed and I. Wolff, Equivalent capacitances of CPW discontinuities and interdigitated capacitors using a three-dimensional finite-difference method, IEEE Trans Microwave Theory Tech 38 (1990), 1808-1815.

16. R. Kulke, P. Pogatzki, D. Kother, T. Sporkmann, and I. Wolff, Enhancement of coplanar capacitor models and verification up to $67 \mathrm{GHz}$ for (M)MIC circuit design, Proc $24^{\text {th }}$ Europ Microwave Conf, Cannes, 1994, pp. $258-262$.

17. R. Kulke, T. Sporkmann, D. Kother, I. Wolff, and P.
Pogatzki, Modeling and analysis aided coplanar design, Microwaves RF (1995), 89-96.

18. Q.J. Zhang and K.C. Gupta, Neural networks for RF and microwave design, Artech House, Boston, 2000.

19. C. Yildiz and M. Turkmen, A CAD approach based on artificial neural networks for shielded multilayered coplanar aaveguide, AEUE: Int J Electron and Commun 58,(2004), 1-9.

20. X. Ding, V. Devabhaktuni, B. Chattaraj, M. Yagoub, M. Deo, J. Xu, and Q.-J. Zhang, Neural-network aproaches to electromagnetic-based modeling of passive components and their applications to high-frequency and high-speed nonlinear circuit optimization, IEEE Trans Microwave Theory Tech 52 (2004), 436449.

21. J. Rayas-Sanchez, EM-based optimization of microwave circuits using artificial neural networks: The stateof-the-art, IEEE Trans Microwave Theory Tech 52 (2004), 420-435.

22. R.S. Chen, X. Zhang, K.F. Tsang, and K.N. Yung, Modeling and design of an interdigital capacitor based on neural networks and genetic algorithm, Microwave Opt Technol Lett (2003), 38 231-235.

23. V. Devabhaktuni, C. Xi, F. Wang, and Q.-J. Zhang, Robust training of microwave neural models, Int J RF and Microwave CAE 12 (2002), 109-124.

24. J. Jargon, K.C. Gupta, and D. DeGroot, Applications of artificial neural networks to RF and microwave measurements, Int J RF and Microwave CAE 12 (2002), 3-24.

25. W. Hilberg, From approximations to exact relations for characteristic impedances, IEEE Trans Microwave Theory Tech 17 (1969), 259-265.

26. S. Gevorgian, personal communication.

27. K. Beilenhoff, H. Klingbeil, W. Heinrich, and H. Hartnagel, Open and short circuits in coplanar MMICs, IEEE Trans Microwave Theory Tech 41 (1993), 15341537.

28. M-H. Mao, R.-B. Wu, C.-H. Chen, and C.-H. Lin, Characterization of CPW open-end capacitance: Theory and experiment, IEEE Trans Microwave Theory Tech 42 (1994), 1016-1024.

29. G. Ghione, A CAD-oriented analytical model for the losses of general asymmetric coplanar lines in hybrid and monolithic MICs, IEEE Trans Microwave Theory Tech 41 (1993), 1499-1510.

30. S. Haykin, Neural networks: A comprehensive foundation, Macmillan College Publishing, New York, 1994.

31. M.T. Hagan, H.B. Demuth, and M.H. Beale, Neural network design, PWS Publishing, Boston, 1996.

32. K.C. Gupta, R. Garg, I. Bahl, and P. Bhartia, Microstrip lines and slotlines, $2^{\text {nd }}$ ed., Artech House, 1996, Boston, p. 133.

33. IE3D EM Simulator, Zeland Software, Inc., CA. 


\section{BIOGRAPHIES}

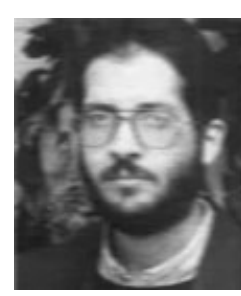

Nihad Dib obtained his B.Sc. and M.Sc. degrees in EE from Kuwait University in 1985 and 1987, respectively. He obtained his $\mathrm{Ph} . \mathrm{D}$. in EE (major in electromagnetics and microwaves) in 1992 from University of Michigan, Ann Arbor and then worked as an Assistant Research Scientist in the radiation laboratory at the same school. In September 1995, he joined the EE department at Jordan University of Science and Technology (JUST) as an Assistant Professor, and became an Associate Professor in September 2000 His research interests are in computational electromagnetics and modeling of planar circuits.

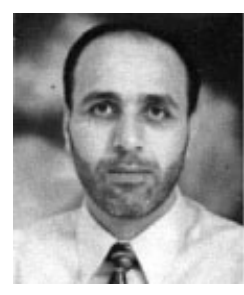

Jehad Ababneh was born in Irbid, Jordan, in 1966. He received his B.S. degree from Jordan University of Science and Technology, Irbid, Jordan, in 1989, his M.E. degree from Tennessee State University, Nashville, TN, in 1998, and his Ph.D. degree from Wichita State University, Wichita, KS, in 2001, all in electrical engineering. He is currently with the electrical engineering department at Jordan University of Science and Technology. His research interests include wireless communication, artificial intelligence, digital signal processing, and speech processing, modeling, and recognition.

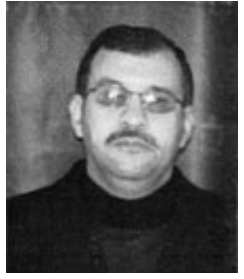

Amjad Omar was born in Kuwait in 1963. $\mathrm{He}$ received his B.Sc. and M.Sc. degrees from Kuwait University in 1985 and 1988, respectively, and his $\mathrm{Ph} . \mathrm{D}$. degree from the University of Waterloo in 1993. He is currently an Assistant Professor at the Hijjawi Faculty of Engineering Technology at Yarmouk University, Jordan. His research interests are in the numerical solution of microwave integrated circuits and antennas. 